\title{
COMMENT
}

\section{Early career investigator highlight: September}

\author{
Amal Isaiah (iD) \\ Pediatric Research (2020) 88:348; https://doi.org/10.1038/s41390-020-1042-z
}

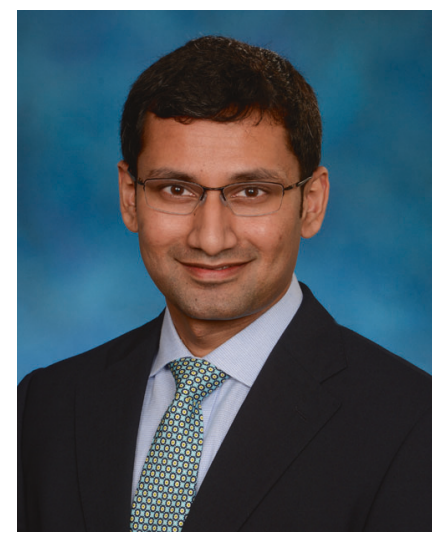

I was born and raised in Cochin, a city in the southern Indian state of Kerala. My parents, both dedicated physicians in my hometown, provided me with the greatest inspiration to continue their line of profession. I received medical education at St. John's Medical College in Bangalore, India and was awarded a Rhodes Scholarship to pursue a D. Phil. in Neuroscience at Oxford University, United Kingdom. At the Auditory Neuroscience Group led by Andrew King, FRS, I developed a cross-modal training paradigm for the improvement of binaural hearing in ferrets fitted with cochlear implants and assessed its impact on the developing brain using electrophysiology and behavior.

As my research interests were translational in nature, I returned to clinical medicine by matching at the University of Maryland for residency training in Otolaryngology. During surgical training, I became interested in the outcomes of tonsillectomy and adenoidectomy (T\&A), a surgical procedure performed in half a million children in the United States each year principally for the management of obstructive sleep disordered breathing (oSDB). Following completion of fellowship training in Pediatric Otolar- yngology at UT Southwestern Medical Center in Dallas, I was recruited back by the University of Maryland School of Medicine (UMSOM) as a faculty member in the Department of Otorhinolaryngology-Head and Neck Surgery.

My principal research interest focuses on understanding neurobehavioral outcomes in children with OSDB utilizing data science. Backed by a superb clinical, educational, and research environment at UMSOM, I have been able to work with many established intramural and extramural researchers who have donated their valuable time for my career development. The current study, ${ }^{1}$ performed in collaboration with Dr. Gautam Das, a well-known computer scientist at the University of Texas Arlington, seeks to improve the risk stratification process for children with OSDB. As polysomnography, the gold standard for the diagnosis and stratification of OSDB, is expensive and resource intensive, our results, from the use of machine learning and wearable physiologic sensors, could support further investigation of a home-based screening pathway for OSDB in children. It is our hope that these efforts will play a role in mitigating the rising healthcare costs associated with diagnosis and management of OSDB, while maintaining the standard of care and predictive accuracy for its screening. My advice to those pursuing similar goals: never be afraid of questioning dogma.

\section{ADDITIONAL INFORMATION}

Competing interests: The author declare no competing interests.

Publisher's note Springer Nature remains neutral with regard to jurisdictional claims in published maps and institutional affiliations.

\section{REFERENCE}

1. Bertoni, D., Sterni, L. M., Pereira, K. D., Das, G. \& Isaiah, A. Predicting polysomnographic severity thresholds in children using machine learning. Pediatr Res. https:// doi.org/10.1038/s41390-020-0944-0 (2020). 